\title{
Effect of urea super granules, prilled urea and poultry manure on the yield of transplant Aman rice varieties
}

\author{
M. H. Kabir, M. A. R. Sarkar and A. K. M. S. H. Chowdhury \\ Department of Agronomy, Bangladesh Agricultural University, Mymensingh-2202, Bangladesh
}

\begin{abstract}
An experiment was carried out at the Agronomy Field Laboratory, Bangladesh Agricultural University, Mymensingh in transplant Aman season 2008 to find out the effect of urea super granules (USG), prilled urea (PU) and poultry manure (PM) on the yield and yield attributes of transplant Aman rice varieties. Two transplant Aman rice varieties viz. BRRI dhan41 and BRRI dhan46 and ten levels of integrated nutrient management encompassing USG, PU and PM were tested following randomized complete block design with three replications. It was observed that variety $\mathrm{BRRI}$ dhan41 gave higher grain yield than BRRI dhan46. For fertilizer effect, the highest grain yield $\left(5.17 \mathrm{t}\right.$ ha $\left.{ }^{-1}\right) \mathrm{was}$ obtained from full dose of USG $(1.8 \mathrm{~g})$ and other inorganic fertilizers, which was similar to that obtained from full dose of USG $(1.8 \mathrm{~g})+\mathrm{PM}$ at $2.5 \mathrm{t} \mathrm{ha}^{-1}$ followed by full dose of PU + PM at $2.5 \mathrm{t} \mathrm{ha}^{-1}$. The lowest grain yield was obtained in the control treatment. In case of interactions, the highest grain yield was obtained in BRRI dhan $41 \times 1.8 \mathrm{~g}$ USG and other inorganic fertilizers which was similar to that of the same dose of USG with PM at $2.5 \mathrm{t} \mathrm{ha}^{-1}$ and the lowest yield was obtained in the control treatment. It was assessed that a considerable portion (31.25\%) of PU nitrogen could be saved by using USG $(1.8 \mathrm{~g})$ together with other inorganic fertilizers or with PM at $2.5 \mathrm{t} \mathrm{ha}^{-1}$.
\end{abstract}

Keywords: Urea super granules, Prilled urea, Poultry manure, Yield, Transplant Aman rice variety

\section{Introduction}

Rice is the major food crop and staple food of Bangladesh. But the average yield of rice is poor in Bangladesh, only $2.6 \mathrm{t} / \mathrm{ha}$ (BBS, 2008). On the other hand, rice production area is decreasing day by day due to high population pressure. The possibility of horizontal expansion of rice production area has come to a stand still (Hamid, 1991). Therefore, attempts should be taken to increase the yield per unit area. For vertical expansion, the use of modern production technologies should be included, among which high yielding varieties and fertilizer management are remarkable ones.

Variety is the most important factor in rice production. Selection of potential variety, planting in appropriate method and application of optimum amount of nutrient elements, can play an important role in increasing yield and national income.

According to Crasswell and De Datta (1980), broadcast application of urea on the surface soil causes losses upto $50 \%$ but point placement of Urea super granules (USG) at $10 \mathrm{~cm}$ depth may result in negligible loss. USG is a fertilizer that can be applied in the rice root zone at $8-10 \mathrm{~cm}$ depth of soil (reduced zone of rice soil) which can save $30 \%$ nitrogen than prilled urea, increase absorption rate, improve soil health and ultimately increase the rice yield (Savant et al., 1991). The recent literatures on nitrogen use efficiency of rice, in general, would indicate the superiority of root zone placement of USG as it would reduce the magnitude of nitrogen losses to a considerable extent and increase its use efficiency for better grain production (Crasswell and De Datta , 1980; Pillai, 1981).

Almost all soils of Bangladesh are deficient in nitrogen mainly due to low level of organic matter caused by rapid decomposition due to warm climate, continuous intensive cropping, cultivation of high yielding varieties and no adding of organic matter. Most of the soils of Bangladesh have less than $1.5 \%$ and in some cases less than $1 \%$ organic matter. Poultry manure (PM) may play a vital role in soil fertility and productivity improvement thereby reducing the use of chemical fertilizers and environmental pollution.

It is true that sustainable production of crops can not be maintained by using only chemical fertilizers and similarly it is not possible to obtain higher crop yield by using organic manure alone (Bair, 1990). In near future, fertilizer $\mathrm{N}$ is likely to be even more costly. This situation in turn will pose a serious threat to food security for the vast millions of people of this country. The use of PM and its proper management may reduce the need for chemical fertilizer allowing the small farmer $s$ to save part of their cost of crop production. The present study was, therefore, carried out to observe the integrated nutrient management by using USG, PU and PM on the yield of transplant Aman rice. 


\section{Materials and Methods}

The experiment was carried out at the Agronomy Field Laboratory, Bangladesh Agricultural University, Mymensingh in transplant Aman season 2008. The experiment consisted of two transplanted Aman rice varieties viz. $V_{1}=B R R I$ dhan4 1 and $V_{2}=B R R I$ dhan46; and ten levels of integrated nutrient management viz. $\mathrm{T}_{1}=$ Control (No PM and NPKSZn fertilizers), $\mathrm{T}_{2}=\mathrm{PM}$ at $5 \mathrm{t} \mathrm{ha}{ }^{-1}, \mathrm{~T}_{3}=\mathrm{PM}$ at $2.5 \mathrm{t} \mathrm{ha-1}, \mathrm{T}_{4}=$ Recommended dose of $\mathrm{PU}$ and other inorganic fertilizers (i.e. 80,60,40,10,5 kg of N, $\mathrm{P}_{2} \mathrm{O}_{5}, \mathrm{~K}_{2} \mathrm{O}, \mathrm{S}$, $\mathrm{ZnSO}_{4}$, respectively ha ${ }^{-1}$ ), $\mathrm{T}_{5}=$ Full dose of USG and other inorganic fertilizers (i.e. $55,60,40,10,5 \mathrm{~kg}$ of N, $\mathrm{P}_{2} \mathrm{O}_{5}, \mathrm{~K}_{2} \mathrm{O}, \mathrm{S}, \mathrm{ZnSO}_{4}$, respectively ha $\left.{ }^{-1}\right), \mathrm{T}_{6}=1 / 2 \mathrm{PU}$ and $\mathrm{PKSZn}+\mathrm{PM}$ at $2.5 \mathrm{tha}^{-1}, \mathrm{~T}_{7}=0.9 \mathrm{~g}$ USG $+\mathrm{PM}$ at $2.5 \mathrm{t} \mathrm{ha}^{-1}, \mathrm{~T}_{8}=0.9 \mathrm{~g} \mathrm{USG}+\mathrm{PM}$ at $5 \mathrm{t} \mathrm{ha}^{-1}, \mathrm{~T}_{9}=1.8 \mathrm{~g}$ USG (full dose for transplant Aman) $+\mathrm{PM}$ at $2.5 \mathrm{t}$ $\mathrm{ha}^{-1}$ and $\mathrm{T}_{10}=$ Full dose of PU (for transplant Aman) + PM at $2.5 \mathrm{t} \mathrm{ha}^{-1}$. The experiment was laid out in a randomized complete block design with three replications. The unit plot size of the experiment was $4.0 \mathrm{~m}$ $\times 2.5 \mathrm{~m}$. Thirty- day old seedlings were transplanted at spacing of $20 \mathrm{~cm} \times 20 \mathrm{~cm}$ with two seedlings hill ${ }^{-1}$. All the fertilizers except prilled urea were applied as basal at final land preparation. Prilled urea was top dressed in three equal splits at 15, 30 and 45 days after transplanting (DAT). Gap filling, weeding, irrigation and other necessary intercultural operations were done in proper time. The crops were harvested at full maturity. The maturity of crops was determined when some $80 \%$ of the grains became golden yellow in colour. Five sample plants were randomly selected and uprooted prior to harvesting from each plot excluding border rows to record data on yield contributing characters. Central $5 \mathrm{~m}^{2}$ area in each plot was harvested to record grain and straw yields. Grain and straw yields plot $^{-1}$ were recorded after threshing by a pedal thresher, winnowing and sun drying properly. The yield values were adjusted to $12 \%$ moisture content and converted to $\mathrm{tha}^{-1}$. All the collected data were analyzed following standard statistical procedure and differences among treatment means were adjudged by Duncan's Multiple Range Test (DMRT) (Gomez and Gomez, 1984).

\section{Results and Discussion}

\section{Varietal performance}

In case of varietal effect plant height, total tillers hill ${ }^{-1}$, effective tillers hill ${ }^{-1}$, length of panicle, grains panicle $^{-1}$, unfilled spikelets panicle ${ }^{-1}$, grain yield, straw yield and harvest index were significantly influenced at different levels of significance. Variety BRRI dhan41 produced higher grain and straw yield and harvest index than that of BRRI dhan46 (Table 1). Higher grain yield in BRRI dhan41was due mainly to higher of effective tillers hill ${ }^{-1}$ and grains panicle ${ }^{-1}$.

Table 1. Effect of variety on yield and yield contributing characters of transplant Aman rice

\begin{tabular}{|c|c|c|c|c|c|c|c|c|c|c|c|c|}
\hline Variety & $\begin{array}{l}\text { Plant } \\
\text { height } \\
\text { (cm) }\end{array}$ & $\begin{array}{c}\text { Total } \\
\text { tillers hill-1 } \\
\text { (No.) }\end{array}$ & $\begin{array}{l}\text { Effective } \\
\text { tillers hill-1-1 } \\
\text { (No.) }\end{array}$ & $\begin{array}{c}\text { Non- } \\
\text { effective } \\
\text { tillers hill-1 } \\
\text { (No.) }\end{array}$ & $\begin{array}{l}\text { Length of } \\
\text { panicle } \\
\text { (cm) }\end{array}$ & $\begin{array}{c}\text { Grains } \\
\text { panicle-1 } \\
\text { (No.) }\end{array}$ & $\begin{array}{c}\text { Unfilled } \\
\text { spikelets } \\
\text { panicle-1 } \\
\text { (No.) }\end{array}$ & $\begin{array}{l}1000- \\
\text { grain wt } \\
\text { (g) }\end{array}$ & $\begin{array}{l}\text { Grain } \\
\text { yield } \\
\text { (t ha-1) }\end{array}$ & $\begin{array}{l}\text { Straw } \\
\text { yield } \\
\left(\mathrm{t} \mathrm{ha}^{-1}\right)\end{array}$ & $\begin{array}{c}\text { Biological } \\
\text { yield } \\
\text { (t ha-1) }\end{array}$ & $\begin{array}{c}\text { Harvest } \\
\text { index } \\
(\%)\end{array}$ \\
\hline V1 & 120.60 & 10.80 & 6.13 & 4.67 & 24.90 & 118.02 & 14.74 & 25.94 & 4.39 & 5.38 & 9.77 & 44.84 \\
\hline V2 & 118.60 & 10.16 & 5.66 & 4.50 & 23.20 & 110.96 & 11.82 & 26.03 & 4.28 & 5.53 & 9.81 & 43.40 \\
\hline $\mathbf{s}_{x}^{-}$ & 0.56 & 0.19 & 0.12 & 0.11 & 0.41 & 0.63 & 0.31 & 0.05 & 0.03 & 0.04 & 0.05 & 0.24 \\
\hline $\begin{array}{c}\text { Level of } \\
\text { significance }\end{array}$ & 0.05 & 0.05 & 0.01 & NS & 0.05 & 0.01 & 0.01 & NS & 0.01 & 0.01 & NS & 0.05 \\
\hline CV (\%) & 2.56 & 9.79 & 10.85 & 13.14 & 9.23 & 2.99 & 12.93 & 1.01 & 3.98 & 3.71 & 2.89 & 3.02 \\
\hline
\end{tabular}

In a column, figures with same letter(s) or without letter(s) do not differ significantly whereas figures with dissimilar letter(s) differ significantly as per DMRT, NS = Not significant

$\mathrm{V} 1=\mathrm{BRRI}$ dhan41 and V2= BRRI dhan46

\section{Integrated nutrient management}

For fertilizer effect all the parameters were significant except non-effective tillers hill $^{-1}$, length of panicle, 1000 -grain weight and biological yield. The highest plant height $(122.26 \mathrm{~cm})$ was found from recommended dose of PU with other inorganic fertilizers and the lowest one $(113.37 \mathrm{~cm}$ ) was found from control treatment (Table 2). Similar results were reported by IRRI (1975). The highest grain yield obtained from full dose of USG $(1.8 \mathrm{~g})$ with other inorganic fertilizers $\left(T_{5}\right)$ while the lowest grain yield $\left(3.04 \mathrm{t} \mathrm{ha}^{-1}\right)$ was obtained from control treatment. However, full dose of USG+PM at $2.5 \mathrm{tha}^{-1}$ was as good as $T_{5}$. 
Encouraging results of PM in combination with chemical fertilizers regarding yield improvement of transplant Aman rice have been reported elsewhere (Hasan et al., 2004, Sarkar et al., 2007). The highest straw yield $\left(6.13 \mathrm{t} \mathrm{ha}^{-1}\right)$ was obtained in full dose of USG $(1.8 \mathrm{~g})$ with PM at $2.5 \mathrm{tha}^{-1}$ and the lowest one was found in control treatment. The highest harvest index $(46.78 \%)$ was found from full dose of USG $(1.8 \mathrm{~g})$ with other inorganic fertilizers while the lowest $(41.31 \%)$ one was obtained from PM at $5 \mathrm{t} \mathrm{ha}^{-1}$ (Table 2).

Table 2. Effect of fertilizer on yield and yield contributing characters of transplant Aman rice

\begin{tabular}{|c|c|c|c|c|c|c|c|c|c|c|c|c|}
\hline Fertilizer & $\begin{array}{l}\text { Plant } \\
\text { height } \\
\text { (cm) }\end{array}$ & $\begin{array}{c}\text { Total tillers } \\
\text { hill }^{-1} \text { (No.) }\end{array}$ & $\begin{array}{l}\text { Effective } \\
\text { tillers hill-1 } \\
\text { (No.) }\end{array}$ & 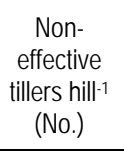 & $\begin{array}{l}\text { Length of } \\
\text { panicle } \\
(\mathrm{cm})\end{array}$ & $\begin{array}{c}\text { Grains } \\
\text { panicle-1 }^{-1} \\
\text { (No.) }\end{array}$ & $\begin{array}{c}\text { Unfilled } \\
\text { spikelets } \\
\text { panicle-1 } \\
\text { (No.) }\end{array}$ & $\begin{array}{l}1000- \\
\text { grain wt } \\
\text { (g) }\end{array}$ & $\begin{array}{l}\text { Grain } \\
\text { yield } \\
\left(\mathrm{t} \mathrm{ha}^{-1}\right)\end{array}$ & $\begin{array}{c}\text { Straw } \\
\text { yield } \\
\left(\mathrm{t} \mathrm{ha}^{-1}\right)\end{array}$ & $\begin{array}{l}\text { Biological } \\
\text { yield } \\
\left(\mathrm{t} \mathrm{ha}^{-1}\right)\end{array}$ & $\begin{array}{c}\text { Harvest } \\
\text { index } \\
(\%)\end{array}$ \\
\hline $\mathrm{T} 1$ & $113.37 \mathrm{c}$ & $7.96 \mathrm{~d}$ & $3.29 d$ & 4.67 & 21.70 & $85.99 i$ & $19.44 a$ & 25.64 & $3.04 f$ & $4.27 \mathrm{~g}$ & 7.31 & $41.43 e$ \\
\hline $\mathrm{T} 2$ & $115.88 \mathrm{bc}$ & $9.34 \mathrm{c}$ & $4.72 \mathrm{c}$ & 4.63 & 23.93 & $103.95 \mathrm{~h}$ & $14.36 \mathrm{~b}$ & 25.84 & $3.49 \mathrm{e}$ & $4.97 f$ & 8.47 & $41.31 \mathrm{e}$ \\
\hline T3 & 119.05ab & $9.61 \mathrm{c}$ & $5.18 b c$ & 4.43 & 24.35 & $108.77 \mathrm{~g}$ & $14.28 b$ & 25.72 & $4.16 \mathrm{~d}$ & $5.22 \mathrm{e}$ & 9.37 & $44.36 \mathrm{bcd}$ \\
\hline T4 & $122.26 \mathrm{a}$ & $10.43 \mathrm{bc}$ & $5.42 \mathrm{bc}$ & 5.01 & 23.95 & $110.05 \mathrm{fg}$ & $15.01 \mathrm{~b}$ & 25.90 & $4.22 \mathrm{~d}$ & $5.46 \mathrm{de}$ & 9.68 & $43.61 \mathrm{~cd}$ \\
\hline T5 & $121.88 \mathrm{a}$ & $12.17 \mathrm{a}$ & $7.51 \mathrm{a}$ & 4.65 & 24.42 & $135.12 \mathrm{a}$ & $8.43 e$ & 26.07 & $5.17 a$ & $5.89 \mathrm{~b}$ & 11.06 & $46.78 a$ \\
\hline T6 & $121.65 a$ & $10.43 \mathrm{bc}$ & $5.97 \mathrm{~b}$ & 4.47 & 24.33 & $113.60 \mathrm{ef}$ & $12.98 \mathrm{bc}$ & 26.21 & $4.50 \mathrm{c}$ & 5.41de & 9.91 & 45.43ab \\
\hline $\mathrm{T} 7$ & $121.98 \mathrm{a}$ & $10.60 \mathrm{bc}$ & $5.56 \mathrm{~b}$ & 5.04 & 24.63 & $114.96 \mathrm{de}$ & $13.96 \mathrm{~b}$ & 26.26 & $4.30 \mathrm{~cd}$ & $5.61 \mathrm{~cd}$ & 9.91 & $43.40 \mathrm{~d}$ \\
\hline T8 & $120.49 \mathrm{a}$ & $10.50 \mathrm{bc}$ & $5.74 b$ & 4.76 & 24.27 & $118.52 d$ & $12.83 \mathrm{bc}$ & 26.19 & $4.50 \mathrm{c}$ & $5.72 \mathrm{bc}$ & 10.23 & $44.00 \mathrm{bcd}$ \\
\hline T9 & 118.17ab & $12.15 a$ & $8.15 a$ & 4.00 & 24.42 & $130.04 b$ & 9.95de & 26.11 & $5.06 a b$ & $6.13 a$ & 11.19 & $45.22 \mathrm{abc}$ \\
\hline T10 & $121.29 \mathrm{a}$ & 11.59ab & $7.43 a$ & 4.16 & 24.50 & $123.91 \mathrm{c}$ & $11.53 \mathrm{~cd}$ & 25.90 & $4.91 \mathrm{~b}$ & $5.84 \mathrm{bc}$ & 10.76 & 45.66ab \\
\hline$s_{x}^{-}$ & 1.25 & 0.42 & 0.26 & 0.25 & 0.91 & 1.40 & 0.70 & 0.11 & 0.07 & 0.08 & 0.12 & 0.54 \\
\hline $\begin{array}{c}\text { Level of } \\
\text { Significance }\end{array}$ & 0.01 & 0.01 & 0.01 & NS & NS & 0.01 & 0.01 & NS & 0.01 & 0.01 & NS & 0.01 \\
\hline CV (\%) & 2.56 & 9.79 & 10.85 & 13.14 & 9.23 & 2.99 & 12.93 & 1.01 & 3.98 & 3.71 & 2.89 & 3.02 \\
\hline
\end{tabular}

In a column, figures with same letter(s) or without letter(s) do not differ significantly whereas figures with dissimilar letter(s) differ significantly as per DMRT, NS =Not significant.

$\mathrm{T}_{1}=$ Control (No PM and NPKSZn fertilizers)

$\mathrm{T}_{2}=\mathrm{PM}$ at $5 \mathrm{tha}^{-1}$

$\mathrm{T}_{3}=\mathrm{PM}$ at $2.5 \mathrm{tha}^{-1}$

$\mathrm{T}_{4}=$ Recommended dose of $\mathrm{PU}$ and other inorganic fertilizers (i.e. 80,60,40,10,5 kg of N, $\mathrm{P}_{2} \mathrm{O}_{5}, \mathrm{~K}_{2} \mathrm{O}, \mathrm{S}, \mathrm{ZnSO}_{4}$, respectively ha ${ }^{-1}$ ).

$\mathrm{T}_{5}=$ Full dose of USG and other inorganic fertilizers (i.e. $55,60,40,10,5 \mathrm{~kg}$ of N, $\mathrm{P}_{2} \mathrm{O}_{5}, \mathrm{~K}_{2} \mathrm{O}, \mathrm{S}, \mathrm{ZnSO}_{4}$, respectively ha ${ }^{-1}$ ).

$\mathrm{T}_{6}=1 / 2 \mathrm{PU}$ and PKSZn $+\mathrm{PM}$ at $2.5 \mathrm{tha}^{-1}$

$\mathrm{T}_{7}=0.9 \mathrm{~g}$ USG $+\mathrm{PM}$ at $2.5 \mathrm{t} \mathrm{ha}^{-1}$

$\mathrm{T}_{8}=0.9 \mathrm{~g}$ USG $+\mathrm{PM}$ at $5 \mathrm{t} \mathrm{ha}^{-1}$

$\mathrm{T}_{9}=1.8 \mathrm{~g}$ USG (full dose for transplant Aman) $+\mathrm{PM}$ at $2.5 \mathrm{t} \mathrm{ha}^{-1}$

$\mathrm{T}_{1 \mathrm{o}}=$ Full dose of PU for transplant Aman $+\mathrm{PM}$ at $2.5 \mathrm{t} \mathrm{ha}^{-1}$

\section{Interaction of variety and integrated nutrient management}

For interaction effect, except total tillers hill ${ }^{-1}$, length of panicle and 1000-grain weight all other parameters were significantly influenced at different levels of significance. The highest effective tillers hill $^{-1}(8.663)$ was found in the BRRI dhan $41 \times 1.8 \mathrm{~g}$ USG with other inorganic fertilizers and the lowest one (3.063) was found in control treatment (Table 3). The highest grain yield $\left(5.27 \mathrm{t} \mathrm{ha}^{-1}\right)$ was obtained in BRRI dhan $41 \times 1.8 \mathrm{~g}$ USG with other inorganic fertilizers, which was as good as the same dose of USG with PM at $2.5 \mathrm{t} \mathrm{ha}^{-1}$. The lowest grain yield was obtained from control treatment. The inefficiency of nitrogen use was attributed to various losses such as ammonia volatilization, denitrification, leaching and runoff (Crasswel and Vlek, 1979). It was also observed in the present study that about $31.25 \%$ of PU nitrogen could be saved by using full dose of USG (1.8g) with other inorganic fertilizers (PKSZn) or full dose of USG (1.8g) with PM at $2.5 \mathrm{t} \mathrm{ha}^{-1}$ in BRRI Dhan41 production. Enhancing results of PM in combination with chemical fertilizers regarding yield improvement of transplant Aman rice have been reported elsewhere (Hasan et al., 2004). The highest straw yield was obtained in both BRRI dhan41 and BRRI dhan46 in combination with $1.8 \mathrm{~g}$ USG and PM at $2.5 \mathrm{t} \mathrm{ha}^{-1}$. However, BRRI dhan $46 \times 1.8 \mathrm{~g}$ USG with other inorganic fertilizers and BRRI dhan $41 \times$ full dose of PU and PM at $2.5 \mathrm{t} \mathrm{ha}^{-1}$ were similar regarding straw yield. The lowest straw yield was obtained in control treatment. 
Table 3. Interaction effect of variety and fertilizer on yield and yield contributing characters of transplant Aman rice

\begin{tabular}{|c|c|c|c|c|c|c|c|c|c|c|c|c|}
\hline $\begin{array}{l}\text { Interaction } \\
\qquad(\mathrm{V} \times \mathrm{T})\end{array}$ & $\begin{array}{l}\text { Plant height } \\
\quad(\mathrm{cm})\end{array}$ & $\begin{array}{l}\text { Total tillers } \\
\text { hill }^{-1} \text { (No.) }\end{array}$ & $\begin{array}{l}\text { Effective } \\
\text { tillers hill }^{-1} \\
\text { (No.) } \\
\end{array}$ & $\begin{array}{c}\text { Non-effective } \\
\text { tillers hill } \\
\text { (No.) }\end{array}$ & $\begin{array}{l}\text { Length of } \\
\text { panicle } \\
(\mathrm{cm})\end{array}$ & $\begin{array}{c}\text { Grains } \\
\text { panicle }^{-1} \\
\text { (No.) }\end{array}$ & $\begin{array}{c}\text { Unfilled spikelets } \\
\text { panicle }^{-1} \\
\text { (No.) }\end{array}$ & $\begin{array}{l}\text { 1000- } \\
\text { grain wt } \\
\text { (g) }\end{array}$ & $\begin{array}{c}\text { Grain yield } \\
\qquad\left(\mathrm{t} \mathrm{ha}^{-1}\right)\end{array}$ & $\begin{array}{c}\text { Straw } \\
\text { yield } \\
\left(\mathrm{t} \mathrm{ha}^{-1}\right)\end{array}$ & $\begin{array}{l}\text { Biological } \\
\text { yield } \\
\left(\mathrm{t} \mathrm{ha}^{-1}\right) \\
\end{array}$ & $\begin{array}{c}\text { Harvest } \\
\text { index } \\
(\%) \\
\end{array}$ \\
\hline $\mathrm{V} 1 \times \mathrm{T} 1$ & $111.70 \mathrm{~h}$ & 8.31 & $3.520 \mathrm{~g}$ & 4.787abcd & 25.37 & $94.97 \mathrm{~m}$ & $20.88 a$ & 25.56 & $3.35 \mathrm{~h}$ & $4.22 \mathrm{~h}$ & $7.58 \mathrm{~h}$ & $44.28 \mathrm{bcd}$ \\
\hline $\mathrm{V} 1 \times \mathrm{T} 2$ & 116.4efgh & 10.47 & $5.313 \mathrm{de}$ & $5.153 \mathrm{ab}$ & 24.00 & $102.3 I$ & 18.72ab & 25.74 & $3.58 \mathrm{~h}$ & $4.83 \mathrm{~g}$ & $8.41 \mathrm{~g}$ & $42.68 \mathrm{~cd}$ \\
\hline $\mathrm{V} 1 \times \mathrm{T} 3$ & 116.7defgh & 10.27 & 5.213def & $5.053 \mathrm{ab}$ & 24.33 & 112.6hij & $16.13 \mathrm{bcd}$ & 25.82 & $4.13 \mathrm{~g}$ & $5.12 \mathrm{fg}$ & $9.25 f$ & $44.68 \mathrm{bcd}$ \\
\hline $\mathrm{V} 1 \times \mathrm{T} 4$ & 127.1a & 10.93 & $5.677 \mathrm{de}$ & $5.257 \mathrm{ab}$ & 24.43 & 115.6ghi & $16.52 \mathrm{bcd}$ & 25.86 & $4.16 \mathrm{~g}$ & $5.41 \mathrm{def}$ & 9.57ef & $43.50 \mathrm{bcd}$ \\
\hline $\mathrm{V} 1 \times \mathrm{T} 5$ & 122.7abcd & 12.33 & $8.663 a$ & $3.670 \mathrm{~d}$ & 25.03 & $138.4 a$ & $9.43 \mathrm{ij}$ & 26.08 & $5.27 a$ & 5.83abc & $11.11 \mathrm{a}$ & $47.51 \mathrm{a}$ \\
\hline $\mathrm{V} 1 \times \mathrm{T} 6$ & 125.3ab & 10.93 & $6.337 \mathrm{~cd}$ & 4.597abcd & 25.43 & $118.8 \mathrm{fg}$ & 14.53def & 26.14 & 4.38efg & 5.33ef & 9.71def & $45.11 \mathrm{abc}$ \\
\hline $\mathrm{V} 1 \times \mathrm{T} 7$ & $122.8 \mathrm{abc}$ & 11.13 & $6.120 \mathrm{cde}$ & $5.013 \mathrm{ab}$ & 26.07 & 119.7efg & $15.03 \mathrm{cde}$ & 26.17 & 4.32efg & 5.39ef & 9.710def & $44.49 \mathrm{bcd}$ \\
\hline $\mathrm{V} 1 \times \mathrm{T} 8$ & 121.9abcde & 10.20 & $5.587 \mathrm{de}$ & 4.613abcd & 24.80 & 119.2efg & 13.67defg & 26.14 & $4.50 \mathrm{def}$ & $5.61 \mathrm{bcde}$ & $10.11 \mathrm{~cd}$ & $44.50 \mathrm{bcd}$ \\
\hline $\mathrm{V} 1 \times \mathrm{T} 9$ & 120.1cdefg & 12.07 & 7.757ab & $4.310 \mathrm{bcd}$ & 24.93 & 133.7ab & 10.43ghij & 26.10 & 5.167ab & $6.13 a$ & $11.30 \mathrm{a}$ & 45.72ab \\
\hline $\mathrm{V} 1 \times \mathrm{T} 10$ & $121.4 \mathrm{bcdef}$ & 11.33 & $7.127 \mathrm{bc}$ & $4.207 \mathrm{bcd}$ & 24.60 & 125.1de & 12.03efghi & 25.78 & $5.027 \mathrm{abc}$ & $5.92 \mathrm{ab}$ & $10.94 a b$ & 45.94ab \\
\hline $\mathrm{V} 2 \times \mathrm{T} 1$ & $115.0 \mathrm{gh}$ & 7.61 & $3.063 \mathrm{~g}$ & $4.547 \mathrm{abcd}$ & 18.03 & $77.01 \mathrm{n}$ & 17.99abc & 25.72 & $2.7 \mathrm{i}$ & $4.32 \mathrm{~h}$ & $7.04 \mathrm{i}$ & $38.58 \mathrm{e}$ \\
\hline $\mathrm{V} 2 \times \mathrm{T} 2$ & $115.4 \mathrm{fgh}$ & 8.22 & $4.117 \mathrm{fg}$ & $4.10 \mathrm{bcd}$ & 23.87 & $105.6 \mathrm{kl}$ & 10.00hij & 25.95 & $3.40 \mathrm{~h}$ & $5.11 \mathrm{fg}$ & $8.51 \mathrm{~g}$ & $39.94 \mathrm{e}$ \\
\hline $\mathrm{V} 2 \times \mathrm{T} 3$ & $121.4 \mathrm{bcdef}$ & 8.95 & $5.147 \mathrm{def}$ & $3.80 \mathrm{~cd}$ & 24.37 & $105.0 \mathrm{kl}$ & 12.43efghi & 25.63 & $4.18 \mathrm{fg}$ & $5.31 \mathrm{ef}$ & $9.49 \mathrm{ef}$ & $44.03 \mathrm{bcd}$ \\
\hline $\mathrm{V} 2 \times \mathrm{T} 4$ & 117.4defgh & 9.93 & $5.160 \mathrm{def}$ & 4.77abcd & 23.47 & $104.5 \mathrm{kl}$ & 13.50defg & 25.95 & $4.28 \mathrm{fg}$ & $5.58 \mathrm{cde}$ & $9.79 \mathrm{de}$ & $43.73 \mathrm{bcd}$ \\
\hline $\mathrm{V} 2 \times \mathrm{T} 5$ & 121.1bcdef & 12.00 & $6.367 \mathrm{~cd}$ & $5.63 a$ & 23.80 & $131.9 \mathrm{bc}$ & $7.43 \mathrm{j}$ & 26.07 & $5.07 \mathrm{abc}$ & 5.940ab & 11.01ab & 46.04ab \\
\hline $\mathrm{V} 2 \times \mathrm{T} 6$ & 118.0cdefg & 9.93 & 5.593de & $4.34 \mathrm{bcd}$ & 23.23 & $108.4 \mathrm{jkl}$ & 11.43fghi & 26.27 & 4.62de & $5.48 \mathrm{cdef}$ & $10.11 \mathrm{~cd}$ & $45.75 \mathrm{ab}$ \\
\hline $\mathrm{V} 2 \times \mathrm{T} 7$ & 121.2bcdef & 10.07 & 5.00 ef & $5.07 a b$ & 23.20 & $110.3 \mathrm{ijk}$ & 12.88efgh & 26.35 & $4.28 \mathrm{fg}$ & $5.83 a b c$ & $10.11 \mathrm{~cd}$ & $42.32 d$ \\
\hline $\mathrm{V} 2 \times \mathrm{T} 8$ & 119.1cdefg & 10.80 & $5.887 \mathrm{de}$ & $4.91 \mathrm{abc}$ & 23.73 & 117.8fgh & 12.00efghi & 26.24 & 4.50def & $5.84 a b c$ & $10.34 \mathrm{c}$ & $43.50 \mathrm{bcd}$ \\
\hline $\mathrm{V} 2 \times \mathrm{T} 9$ & 116.3 efgh & 12.23 & $8.547 a$ & $3.68 d$ & 23.90 & $126.3 \mathrm{~cd}$ & 9.47ij & 26.12 & $4.957 \mathrm{bc}$ & $6.13 \mathrm{a}$ & $11.09 a$ & $44.73 \mathrm{bcd}$ \\
\hline $\mathrm{V} 2 \times \mathrm{T} 10$ & 121.2bcdef & 11.85 & 7.740ab & $4.11 \mathrm{bcd}$ & 24.40 & $122.7 \mathrm{def}$ & $11.03 \mathrm{ghi}$ & 26.01 & $4.80 \mathrm{~cd}$ & 5.77abcd & $10.57 \mathrm{bc}$ & 45.39ab \\
\hline $\mathbf{s}_{\mathrm{x}}^{-}$ & 1.77 & 0.59 & 0.37 & 0.35 & 1.28 & 1.98 & 0.99 & 0.15 & 0.10 & 0.12 & 0.16 & 0.77 \\
\hline $\begin{array}{c}\text { Level of } \\
\text { Significance }\end{array}$ & 0.01 & NS & 0.01 & 0.01 & NS & 0.05 & 0.05 & NS & 0.01 & 0.05 & 0.50 & 0.05 \\
\hline CV (\%) & 2.56 & 9.79 & 10.85 & 13.14 & 9.23 & 2.99 & 12.93 & 1.01 & 3.98 & 3.71 & 2.89 & 3.02 \\
\hline
\end{tabular}

In a column, figures with same letter(s) or without letter(s) do not differ significantly whereas figures with dissimilar letter(s) differ significantly as per DMRT.

$\mathrm{V}=$ Variety and $\mathrm{T}=$ Fertilizers, NS $=$ Not significant, $\mathrm{V} 1=\mathrm{BRRI}$ dhan41 and V2= BRRI dhan46, $\mathrm{T}_{1}=$ Control (No PM and NPKSZn fertilizers), $\mathrm{T}_{2}=\mathrm{PM}$ at $5 \mathrm{t}$ ha ${ }^{-1,} \mathrm{~T}_{3}=\mathrm{PM}$ at $2.5 \mathrm{t}$ ha ${ }^{-1,} \mathrm{~T}_{4}=$ Recommended dose of PU and other inorganic fertilizers (i.e. 80,60,40,10,5 kg of N, $\mathrm{P}_{2} \mathrm{O}_{5}, \mathrm{~K}_{2} \mathrm{O}, \mathrm{S}, \mathrm{ZnSO}_{4}$, respectively ha ${ }^{-1}$ ), $\mathrm{T}_{5}=$ Full dose of USG and other inorganic fertilizers (i.e. $55,60,40,10,5 \mathrm{~kg}$ of N$, \mathrm{P}_{2} \mathrm{O}_{5}, \mathrm{~K}_{2} \mathrm{O}, \mathrm{S}, \mathrm{ZnSO}_{4}$, respectively ha $\left.{ }^{-1}\right), \mathrm{T}_{6}=1 / 2 \mathrm{PU}$ and PKSZn $+\mathrm{PM}$ at $2.5 \mathrm{t} \mathrm{ha}^{-1,} \mathrm{~T}_{7}=0.9 \mathrm{~g}$ USG $+\mathrm{PM}$ at $2.5 \mathrm{t} \mathrm{ha}{ }^{-1,} \mathrm{~T}_{8}=0.9 \mathrm{~g}$ USG $+\mathrm{PM}$ at $5 \mathrm{t}$ ha ${ }^{-1,} \mathrm{~T}_{9}=1.8 \mathrm{~g}$ USG (full dose for transplant Aman) + PM at $2.5 \mathrm{t} \mathrm{ha}^{-1,} \mathrm{~T}_{10}=$ Full dose of PU for transplant Aman + PM at $2.5 \mathrm{t} \mathrm{ha}{ }^{-1}$. 
In transplant Aman season, BRRI dhan41 can be successfully cultivated with full dose of USG (1.8g) and other inorganic fertilizers (PKSZn) to obtain the highest grain yield. It is also observed that in transplant Aman season full dose of USG with other inorganic fertilizers (PKSZn) showed the best yield performance.

\section{Acknowledgement}

The financial assistance of the Bangladesh Agricultural Research Council, Farmgate, Dhaka, to carry out the research work is thankfully acknowledged.

\section{References}

BBS (Bangladesh Bureau of Statistics). 2008. Statistical Yearbook of Bangladesh. Bangladesh Bur. Stat., Stat. Div., Mini. Plan. Govt. People's Repub. Bangladesh. Dhaka. p.57.

Bair, W. 1990. Characterization of environment of sustainable agriculture in semi arid tropics. In:Proc. Sustainable Agriculture. Issues, Perspective and Prospects Semi Arid Tropics (Ed. Singh, R, P.) Hydrabad,India. Indian Soc. Agron. pp. 1;90-124.

Craswell, S.K. and S.K. De Datta. 1980. Recent development in research on nitrogen fertilizer for rice. Intl. Rice Res. Institute Research Paper Series No. 119, p. 11.

Crasswell and Vlek. 1979. "Greenhouse Evaluation of Nitrogen Fertilizers for Rice”, Soil Sci. Soc. Am. J., 44: 1184-1188.

Gomez, K.A. and Gomez, A.A. 1984. Statistical Procedures for Agricultural Research. John Willey and Sons, New York. pp. $28-92$.

Hamid, M.A. 1991. A Data Base on Agricultural and Food Grains in Bangladesh (1947-48 to 1989-90). Ayesha Akhtar, 606 North Shahjahanpur, Dhaka, Bangladesh. pp. 30-35.

Hasan, M.K., Sarkar, M.A.R. and Hasan, A.K. 2004. Effect of poultry manure based integrated fertilizer management on growth and yield of aromatic rice. Bangladesh J. Seed Sci. \& Tech. 8(1\&2):97-103.

IRRI (International Rice Research Institute). 1975. Annual Report for 1974, Los Banos, Philippines.

Pillai, K. 1981. Agronomic practices to improve the N use efficiency of rice Fert. News.26(2):3-9.

Sarkar, M.A.R, Rahman, H. and Islam, A.K.M.M. 2007. Effect of combination of poultry manure and inorganic fertilizers on the yield and yield contributing characters of transplant aman rice. Bangladesh J. Crop Sci. 18 (1):7-12.

Savant, N.K., Dhane, S.S. and Talashikar, S.C. 1991. International Fertilizer Development Centre. Muscle, Shoals, Alabama, USA. Fertil. News. 36(3):19-25. 